\title{
Chapter 8: Categories: The Top-Level Ontology
}

Ludger Jansen

The task of ontology is to represent reality or, rather, to support the sciences in their representation of reality. In the last chapter, the reader became acquainted with an important means of doing so, namely: the technique of classification. But, in any classification, what are the very first kinds? What should the top level look like? In this chapter, I attempt to answer these questions. First, I review some suggestions for top-level ontologies with the help of the criteria established in Chapter 7 (section 1). From the point of view of the philosophical tradition of ontology, the question of a top-level ontology is tantamount to the question of the most basic categories. In order to develop some alternative suggestions, the nature of categories must first be addressed. To this end, I appeal to the philosopher whose ideas are pivotal in influencing our current understanding of ontology: Aristotle (section 2). Starting from Aristotle's list of categories (section 3), I go on to discuss three dichotomies which I recommend as candidates for the seminal principles of a top-level ontology, namely: dependent versus independent entities (section 4), continuants versus occurrents (section 5), and universals versus particulars (section 6). Finally, I discuss some categories of more complex entities like states of affairs, sets, and natural classes (section 7).

\section{SUMO, CYC\& Co.}

What should an ontology look like at the highest level? What are the most general classes of all classifications? Authors in the fields of informatics and knowledge representation have offered various suggestions. Some of the best known are:

- the OpenCyc Upper Ontology: the open-source version of the Cyc technology, developed by the Texas-based ontology firm Cycorp, which is supposedly the largest implementation of general knowledge inside a computer for purposes of common-sense reasoning; ${ }^{23}$

${ }^{23}$ See $C y c$, as of August 8, 2006: 'OpenCyc is the open source version of the Cyc technology, the world's largest and most complete general knowledge base and commonsense reasoning engine'. 
- SUMO, the Suggested Upper Merged Ontology, which developed from an open-source project bringing together freely available, noncommercial ontologies into a common system; together with its various domain ontologies SUMO, supposedly, is currently the largest publicly accessible ontology; ${ }^{24}$

- the Sowa Diamond (see Figure 1), representing in graphic form the toplevel ontology suggested by John Sowa, which forms twelve categories by means of two dichotomies and a trichotomy in a lattice-like array (see Figure 1); ${ }^{25}$

- BFO, Basic Formal Ontology, developed by the Institute for Formal Ontology and Medical Information Science (IFOMIS), and which exists in three versions (OWL DL, First-Order Logic, and OBO format). ${ }^{26}$

Figure 1: The Sowa Diamond ${ }^{27}$

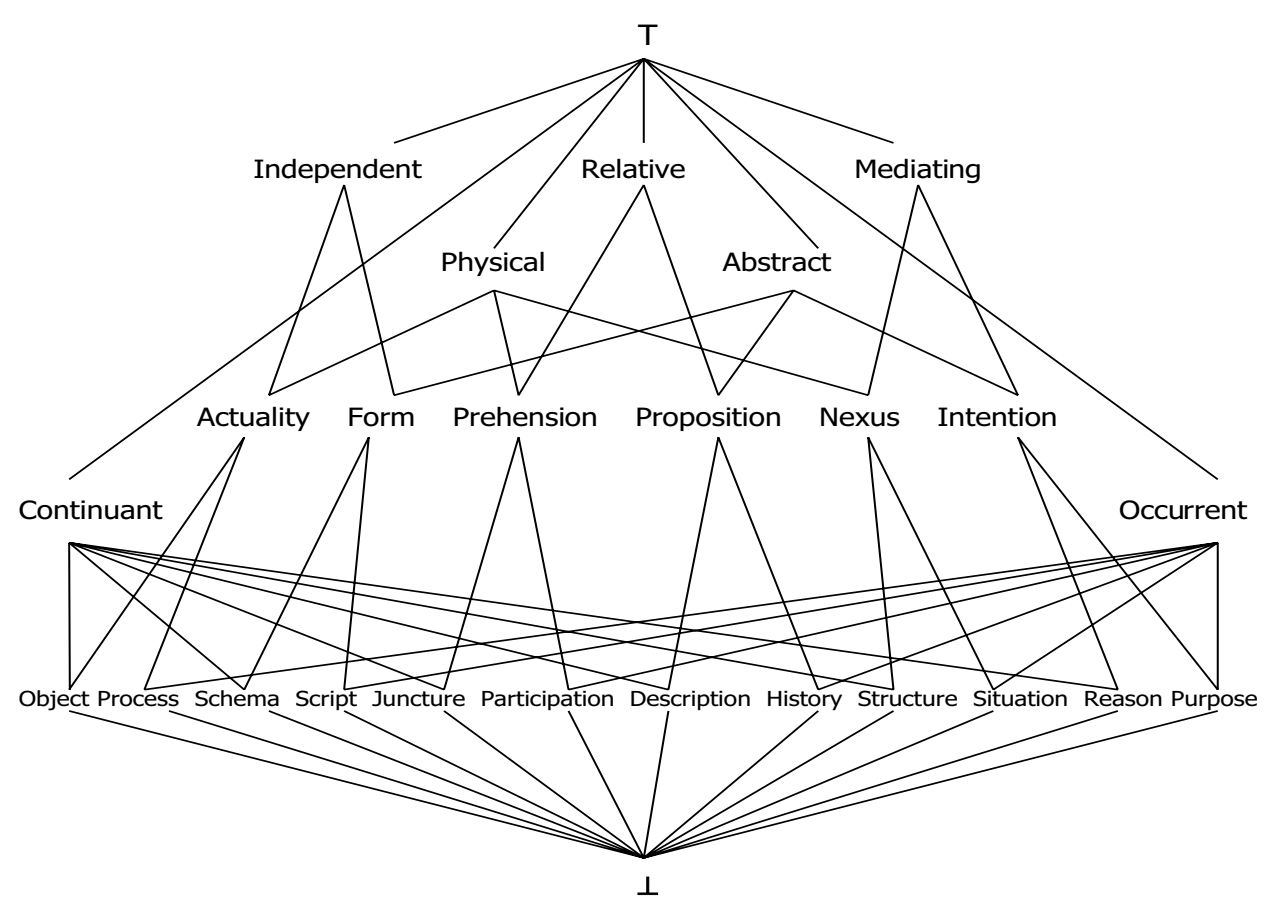

${ }^{24}$ See Ontologyportal, August 8, 2006: 'The Suggested Upper Merged Ontology (SUMO) and its domain ontologies form the largest formal public ontology in existence today'.

${ }^{25}$ Compare Sowa, 2000, 2001.

${ }^{26}$ See BFO; Grenon, et al., 2004; Grenon and Smith, 2004; Grenon, 2003.

${ }^{27}$ Source: John F. Sowa. 'Top-level Categories', http://users.bestweb.net/ sowa/ontolo gy/toplevel.htm (August 8, 2006). 
In the following, I am going to compare OpenCyc to the quality criteria for classifications expounded in the last chapter. The suggestion for an Aristotelian-inspired top-level ontology, which will be developed in what follows, corresponds to the most basic traits of BFO, building on the three dichotomies between independent and dependent entities, continuants and occurrents, and universals and particulars. Over the course of developing these suggestions, it will become clear where the Sowa Diamond needs to be repolished (section 8).

In contrast to the completely symmetrical Sowa Diamond, the top level of the OpenCyc Upper Ontology is a complicated ('tangled') conglomerate. The graphic representation of this classification system in Figure 2 gives us an impression of this.

Against the background of the criteria for classifications addressed in Chapter 7, issues with the highest dichotomy in this diagram become immediately apparent. Why should we divide the class thing into the two subclasses of Individual and PartiallyIntangible? These two classes are neither jointly exhaustive nor pairwise disjoint. The latter, it seems, was introduced to have a place for persons, who putatively embody both tangible and intangible (mind-related) aspects. OpenCyc quite clearly admits of multiple inheritance, which manifests itself in diamond-like structures in the diagram. The reader will notice the combined subclass of PartiallyIntangibleIndividual at the level below these two classes. The two classes mentioned do not exhaust the class of Thing. Non-individuals (that is, the universals) do not appear as such in the diagram. The categories placed in opposition to the Intangible, namely, PartiallyIntangible and TangibleIndividual, do not appear in the diagram until four levels later.

Further, the diagram does not distinguish sufficiently between classificatory differences (such as PartiallyTangible) and the classes thereby engendered (such as TangibleThing). When we read the connective lines in the sense of the is_a relation, as we should be able to do in a classification system, then what results is grammatical nonsense: TangibleThing is_a PartiallyTangible. The subsumption relation is_a does not find application here. An ordinary predicative structure would be much more appropriate here, as in: TangibleThing is PartiallyTangible. 


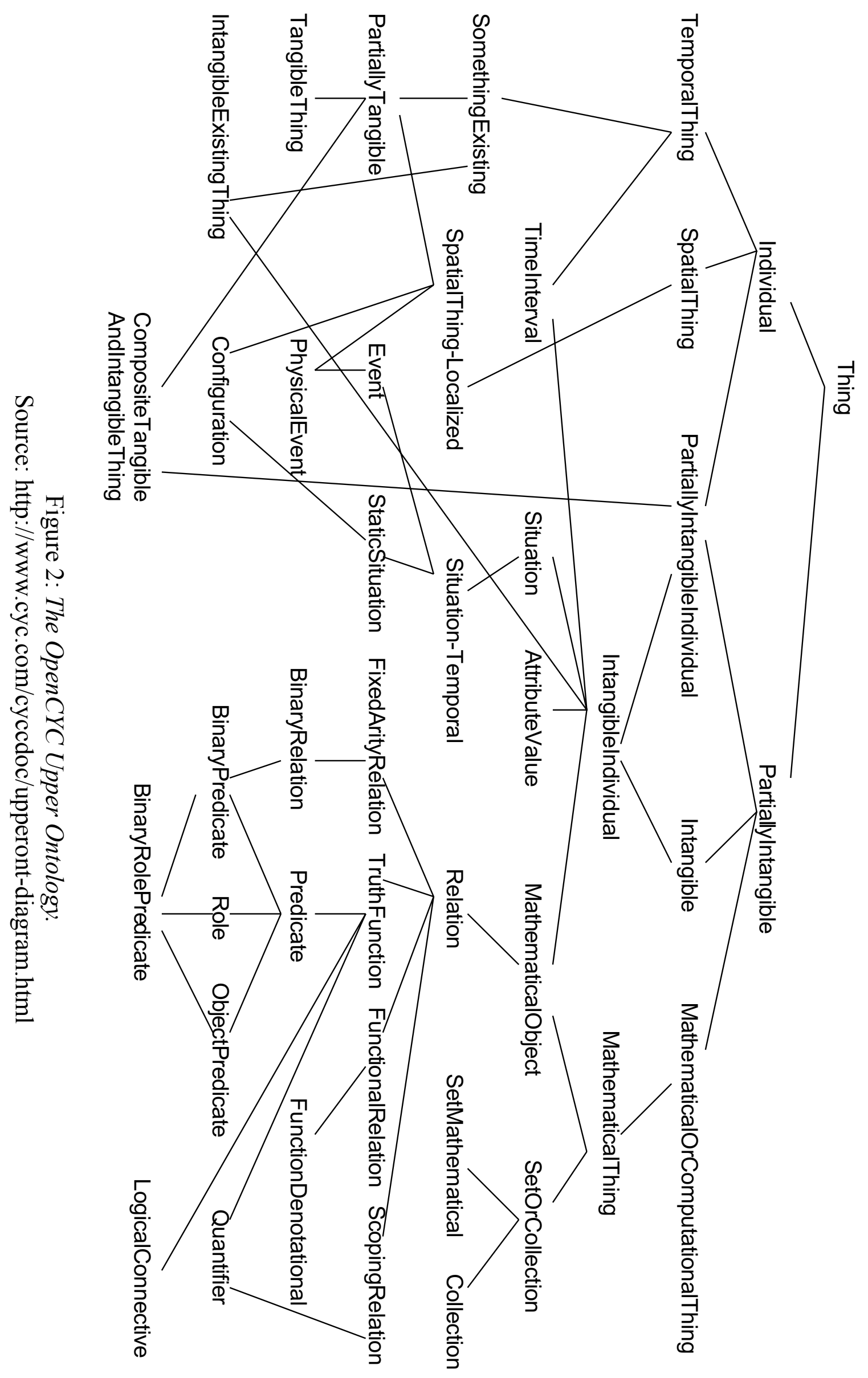


It is surprising that, apart from these problems, the property of tangibility is given such a prominent position in the first place. Attributes such as spatiotemporality or materiality seem to be much more basic and, also, better understood. Like many predicates expressing dispositions, 'tangible' is an extremely ambiguous term. God, an electron, the Milky Way, the Earth's gravitational field, the country of Germany, Beethoven's Fifth Symphony, a sound wave, meanings, neighborliness, freedom, a football game, an hour, yesterday's snow, the exponential function, a computer program, my conception of the moon, and a stone enclosed in epoxide resin are all intangible, but for very different reasons. These reasons indicate aspects of these things that would make better traits on which to base an ontological classification.

OpenCyc's subsumption relations are also problematic with respect to details. TimeInterval is surely a TemporalThing, but is it an Individual? In any case, not in the sense of indivisibility (or more precisely: the inability to be divided into two things of the same kind as the thing divided), for every time interval can be divided into parts which are themselves time intervals. On the other hand, SituationTemporal does indeed seem to be a TemporalThing. The class Relations is subsumed under MathematicalObject. Yet, my being in love with someone, being somebody's neighbor, and being an employee are all relations, but they are not mathematical objects. Similarly, my stamp collection is a Collection, but it is by no means a MathematicalObject, and it is tangible all over; thus, in no way is it a PartiallyIntangible thing.

No ontologically apt classification principles can be found in the diagram's 'or' expressions MathematicalOrComputationalThing and SetOrCollection, for there seems to be no good reason to treat the result of combining two universals by means of an 'or' relation as constituting a universal in its own right (Armstrong, 1978, II, 19-23). The class SomethingExisting is also strange - do the other classes comprehend entities that do not exist? Here the property of existence is wrongly being treated as a characteristic of things (see Frege, 1884, 53, and 1892, 192205). The highly varied division of relations is ultimately based, mainly, on logical considerations; but these are entirely independent of the ontology of relations (see Jansen, 2006).

All of these are good reasons to work towards a more unified and consistent form for the uppermost levels of classification systems appropriate for ontologies. In what follows, such a unified form will be 
developed drawing upon one of the oldest suggestions for such a top-level ontology, namely: Aristotle's Categories.

\section{What are Categories?}

As far as we know, Aristotle was the first to use the Greek word kategoria as a technical term in the context of philosophy. Originally, the noun kategoria and its corresponding verb, katêgorein, belonged to legal discourse. There, kategoria means the accusation in front of the judge, and katêgorein means to accuse someone. Probably because an accusation asserts something of someone, the verb can also mean 'make known' or 'assert', and was used in this way by Plato. ${ }^{28}$ Aristotle uses the active verb phrase kategorein ti tinos in the sense of 'to assert something about something', but even more often he uses the passive katêgoreisthai ti tinos or katêgoreisthai ti kata tinos in the sense of 'is said of something'. Aristotle uses the noun kategoria as the technical term for predication or for the predicate itself. In addition, he uses the plural of the noun in the sortal sense of 'kinds of predicates/of predication', and it is only in this usage that the Greek word kategoria can be translated into English as category (Jansen, 2006).

We have evidence that Aristotle's conception of the categories developed in three phases. First, as in Topics I 9, the distinction of different categories was only meant as a classification of predicates. In this first phase, the categories served as aids for finding arguments and for avoiding or discovering false inferences; thus, they had their place in the theory of argumentation. The second phase is represented in Aristotle's Categories. There the division of categories encompasses, not only predicate terms, but also subject terms. In this phase, terms denoting so-called primary substances, i.e. proper names such as 'Socrates' or 'Brunhilde', fall under the first category of substance, although they can function only as the subject of predication but never as predicates (Categories 5, 3a 36-37). This represents a step away from the theory of argumentation in the direction of ontology. In the third phase, which finds its expression in the Metaphysics, we find Aristotle's famous observation that 'to be' and 'a being' are used in as many different ways as there are categories (Metaphysics V 7, 1017a 22-23). Here, the division into separate categories

${ }^{28}$ See e.g. Plato, Theaetetus 208b; Phaedrus 73b. Theaetetus 167 a links both meanings with each other. 
became a full-fledged part of one of the most important of Aristotle's ontological teachings.

Aristotle's theory of categories was the subject of much dispute in antiquity, and has been interpreted in a variety of ways in the history of philosophy. Partly, this has to do with the fact that category theory had many different facets, even in the works of Aristotle himself. This came about because either Aristotle subjected his ideas to further development, or highlighted different aspects when presenting his theory. We can distinguish four prototypical interpretations (which often appear in combination), according to whether the categories classify (1) subject and predicate terms and the associated meanings, (2) beings, (3) mental or extra-mental concepts, or (4) meanings of the copula 'is'. ${ }^{29}$ Here, we can draw on what was certainly the main conception of the late Aristotle, namely: that of the categories as the highest species of beings.

\section{Aristotle's Ten Categories}

In Topics I 9, Aristotle says explicitly that there are ten categories, which he then proceeds to delineate. A list of ten categories can also be found in the Categories (see Figure 3). Aristotle names many of his categories with the interrogative expressions that one would use to ask questions whose answers would make reference to entities in the respective categories. Many of the current names for these categories have their origins in the corresponding Latin interrogative expressions.

Figure 3: Different Terms for Aristotle's Categories

\begin{tabular}{|c|c|c|c|}
\hline ARISTOTLE'S TERM & ENGLISH TRANSLATION & LATIN TERM & MODERN TERMS \\
\hline ti esti, ousia & What is it?, essence & $\begin{array}{c}\text { quod est, quiditas, } \\
\text { essentia }\end{array}$ & essence \\
\hline poson & How much? & quantum, quantitas & quantum, quantity \\
\hline poion & How is it? & quale, qualitas & quality \\
\hline pros $t i$ & Related to what? & relativum & relative, relation \\
\hline pou & Where? & ubi & place \\
\hline pote & When? & quando & time \\
\hline keisthein & lying, being situated & situ & position, posture \\
\hline echein & having & habitus & \\
\hline poiein & doing & agere & \\
\hline paschein & suffering & pati & \\
\hline
\end{tabular}

${ }^{29}$ See Bonitz, 1853; Ebert, 1985; Kahn, 1978; Oehler, 1986. 
Kant accused Aristotle of choosing his categories in a rhapsodic manner. In this unsystematic way, Aristotle could never be certain that his list of categories was complete (Kant, 1781, A $81=\mathrm{B}$ 106-107). Later Aristotelians, such as Thomas Aquinas ${ }^{30}$ or Franz Brentano (1862; see also Simons, 1992), undertook the task of constructing a system that yields the Aristotelian categories, in the precise order in which they are named and discussed in the Categories. ${ }^{31}$ We can assume that Aristotle himself constructed his list of categories indeed in an unprincipled way, as Kant suspected, for he seems to have proceeded simply on the basis of his experience in dialectical exercises and philosophical discussions.

This might explain the disparity of Aristotle's list of categories, since the elements in his list are not at all of the same standing. There are two important ways in which Aristotle's categories fall into disparate groups, which I will discuss in due course: They encompass dependent as well as independent entities (section 3), and continuants as well as occurrents (section 4). These are already two of the ontological dichotomies that can be used as the seminal principles of the top-level ontology. Following these, I will introduce a third dichotomy that is orthogonal to the other two: the distinction between universals and particulars (section 5).

\section{Dependent and Independent Entities}

In the Categories, Aristotle distinguishes between primary substance (protê ousia), that is, a substantial particular, and secondary substance (deutera ousia), a species of substantial particulars. Of these two, Aristotle accords special ontological status to the individual substances. Everything else is either predicated of these individual substances, or is in them as something underlying them (Categories 5. 2a 34-35; 2b 3-5; 2b 15-17). In later texts as well, Aristotle accords this first category of individual substance a special importance with respect to the other categories, which are also called 'affections of the ousia'. ${ }^{32}$ Aristotle is quite clear that his ten categories are not to be viewed as equals; rather, the individual substances

${ }^{30}$ See Aquinas, In Physicorum Aristotelis expositio III, lectio 5, Nr. 322 [15] and In Metaphysicorum Aristotelis expositio V, lectio 9, Nr. 891-892.

${ }^{31}$ See Jansen 2007 for a new suggestion of a hierarchy of Aristotle's categories along the lines suggested here.

${ }^{32}$ Metaphysics IV 2, 1003b6: ousiai - pathê ousias; see also Metaphysics XIV 2, 1089b 23: ousiai-pathê-pros ti. 
are presupposed by the other categories. From Aristotle's perspective, it is this fact that made the unity of ontology possible (Metaphysics IV 2).

Customarily, the dependent categories are called accidents and are placed in opposition to substances. A traditional criterion for the opposition of substances and accidents can be found in the second chapter of the Categories: qualities and quantities are in a substance, while substances are not in a substance but, rather, are identical with one. But it is not entirely clear how this 'being in something else' is to be understood; for a heart is in a body and a tapeworm is in a host. This could not be the type of 'being in something else' that Aristotle meant. Aristotle explicitly excludes 'being-in' in the sense in which a part is in a whole as the heart is in the body. But a parasite such as a tapeworm is not a part of its host.

The criterion of ontological dependence helps to solve this problem. The tapeworm could leave its host and move into another host. A grin, a certain height, or a certain color could not leave their bearers in this way and continue to exist. It is not possible for the Cheshire Cat to disappear and leave its grin behind. ${ }^{33}$ The height of a tree cannot continue to exist when the tree is destroyed. The color of a test tube cannot remain in a room when the test tube is taken out of the room. The grin, the height, and the color are dependent for their existence upon a bearer, a substance which has this grin, this height, or this color, among its properties. They cannot migrate from this substance to another: if Alice were to grin instead of the Cheshire Cat, then it would be a new grin.

Let us summarize this thought. Substances do not need the entities of other categories in order to exist, whereas the entities of other categories require entities from the first category for their existence. For this reason, substances are called ontologically independent entities, where accidents are said to be ontologically dependent. More precisely: substances are ontologically independent of accidents, while accidents are ontologically dependent upon substances. The notion of ontological dependence can be formally captured through a counterfactual criterion:

Def. (6.1) An entity $x$ is ontologically dependent upon an entity $y$ if $x$ could not exist if $y$ did not exist.

${ }^{33}$ Lewis Carroll, Alice's Adventures in Wonderland, Chapter 6: 'I've often seen a cat without a grin, thought Alice; but a grin without a cat! It's the most curious thing I ever saw in my life!' (Carroll, 1965, 67). 
For substances and their accidents it holds that: if $s$ is a substance and $a$ is one of $s$ 's accidents, then $a$ cannot exist unless $s$ exists. Because $a$ inheres in $s, a$ is ontologically dependent upon $s$. On the other hand, however, not all of those things that are ontologically dependent on other entities inhere in those entities. A relational event such as a kiss or a hit are ontologically dependent upon their relata, but they do not of inhere in any of their relata; rather, they inhere in the totality which these relata form.

It is possible for two entities to be mutually ontologically dependent. Someone can only be a patient when there is a doctor treating him, and there can only be an active doctor when there is also a patient. Now, being a doctor is not dependent upon the existence of a particular individual patient; any patient, at all, would be sufficient. By the same token, the existence of patients does not end when a single individual doctor ceases to exist. Only if there are no more doctors whatsoever can there be no more patients. Doctors and patients are thus generically dependent upon one another. We can define generic dependence as:

(Def. 6.2) Being $F$ is generically dependent upon being $G$ if nothing can be $F$ unless something is $G$.

On this definition, generic ontological dependence is a relation between universals.

We had defined ontological dependence in such a way that it is a relation that could obtain, in principle, between entities in any category; thus ontological dependence can also obtain between universals, according to the following definition:

(Def. 6.3) A universal $F$ is ontologically dependent upon a universal $G$ if the universal $F$ cannot exist unless universal $G$ exists.

The best criterion for determining whether the existence of a universal $F$ presupposes the universal $G$, is to ask whether $F$ could exist if nothing at all is $G$, and this is precisely the definition of generic dependence. Hence, there is no difference between the generic dependence of being $F$ on being $G$, and the ontological dependence of the universal $F$ on the universal $G$.

The group of accidents can be further divided into relational and nonrelational entities. Relational entities are those that are ontologically dependent on multiple bearers, while non-relational entities are those that are ontologically dependent upon one bearer only (see Jansen, 2006; Smith and Ceusters, 2007). 


\section{Continuants and Occurrents}

There is another way in which Aristotle's list of categories is not uniform. Two of the Aristotelian categories, those of action and passion, differ in an important way from the others. Whereas a substance such as a bacterium, a quantity such as a length of 20 meters, or a quality such as red, exist in toto at every point in time at which they exist at all, the existence of actions and passions is spread out over the course of some time interval. Whenever we encounter a bacterium, we encounter the whole bacterium at each point in time over the course of the bacterium's life. The process by which a bacterium reproduces, by contrast, or a process such as healing, take place within time and are manifested over a time span. The process of reproduction has a beginning and an end; it is composed of various phases that follow one another in time. These entities, reproduction and healing, have temporal parts. By contrast, the bacterium has spatial parts - for example, a nucleus, a membrane, and a cytoplasm - which exist at one and the same time.

Hence, we see that there are two kinds of entities that stand in intimate relation to one another, namely: (1) an organism and (2) its life or history (which might be documented in a patient record). The organism itself is present as a whole at every point of its existence, while the life of the organism is spread out over multiple points in time. In the former case we are dealing with entities which continue to exist through time, which we call continuants. In the latter case, by contrast, there is no point of its existence at which the entity is wholly present. It unfolds in time, that is, it has temporal stages or phases. The latter are not identical with one another, but are rather various different parts of the temporal entity. These are things that occur in time, and for this reason are called occurrents.

The words 'continuant' and 'occurrent' can be traced back to the Cambridge logician William Johnson (the teacher of Bertrand Russell). Johnson defines 'continuant' as 'that which continues to exist while its states or relations may be changing' $(1921,199)$. More recently, David Lewis $(1986,202)$ drew a similar distinction between endurers and perdurers:

Something perdures iff it persists by having different temporal parts, or stages, at different times, though no one part of it is wholly present at more than one time; whereas it endures iff it persists by being wholly present at more than one time. 
Distinguishing between these two modes of existence is often seen as marking a distinction between two alternative, and competing, theories of the diachronic behavior of the same entities. David Lewis, for example, claimed that all entities must be seen as four-dimensional perdurers (thus as occurrents). ${ }^{34}$ Here, instead, we will argue that Socrates and his walking exhibit two very different modes of existence. While the walking is clearly an occurrent, Socrates himself is no less clearly a three-dimensional continuant. Hence, there are two kinds of entities which demand distinct theories to account for their diachronic behavior. We need both continuants and occurrents in order to represent reality accurately.

But the opposition between continuants and occurrents does not present an exhaustive classification of all entities. For this opposition appears only with those entities whose existence, in fact, is extended over multiple points in time. There are at least two problem cases which this distinction does not encompass, namely, instantaneously existing qualities and quantities (see Johansson, 2005), and points in time themselves. It is trivially true that a point in time exists only at one point in time, that is, at itself. And, in processes of growth and change, it is possible for instantaneously existing quantitative and qualitative individuals to be substituted for each other. If a ball grows continuously at a constant rate during this growth process there are no two points at which the ball has the same weight. If a surface changes its color continuously from, say, blue to red, at no two points in time is this surface the same color. Since the existence of these instantaneous qualities and quantities does not extend over multiple points in time, it would seem to follow that there are qualities and quantities which fall under the category of continuant, as well as those which do not. In the same way, time intervals would belong to the category of occurrent, but points in time would not. This does not make for a particularly elegant theory. So, we will modify these categories slightly, in order to integrate these homeless entities.

If we picture the world at any single point in time, we will discover people, animals, artifacts, colors, sizes, and relations in our picture. But changes, processes, and events that are taking place at that point in time will not be visible in the picture. In order to represent these, we need a sequence of pictures instead of a single picture; we need a film. In order to obtain a complete picture of our ever changing world, we thus need two kinds of representation.

\footnotetext{
${ }^{34}$ For an overview of this discussion, see e.g. Lowe, 2002, 49-58.
} 
On the one hand, we need snapshots of the world at particular points in time, which capture the continuants. Let us call such snapshots SNAP ontologies (following Grenon and Smith, 2004). Included among SNAP entities are substances, quantities, qualities, relations, as well as the boundaries of substances, collections of substances, places such as niches and holes, and spatial regions such as points, lines, surfaces, and volumes. Over and above to the traditional category of continuants, SNAP ontologies comprise also the merely instantaneously existing instances of qualities and quantities which would otherwise be ontologically homeless.

On the other hand, we need a representation of change, something like a film which represents entire time spans. We will call these SPAN ontologies (after Grenon and Smith, 2004). Included among SPAN entities are happenings such as processes and events, temporal regions such as time intervals with time points as their boundaries, as well as spatiotemporal regions. In Chapter 12 we will discuss happenings, the specific elements of SPAN ontologies. Time points, in spite of their lack of temporal extension, belong to the SPAN ontology and not to the SNAP ontology. A single SNAP ontology, which represents the world at a given point in time, is linked to this time point as to its date, but does not contain this time point as one of the entities in its coverage domain.

\section{Universals and Particulars}

In addition to the two ontological dichotomies already discussed independent vs. dependent entities, continuants vs. occurrents - there is also a third: that between universals and particulars. Since this distinction cuts straight through all of the Aristotelian categories, we can call it transcategorical. ${ }^{35}$ This third distinction is also given systematic treatment in Aristotle's Categories. In the second chapter, he distinguishes between what can and what cannot be predicated of another entity. Predication requires an aspect of generality. Particulars, such as Socrates or my height, cannot be attributed to other entities. Sentences that contain as predicates the expressions 'is Cicero' or 'is my height' are not predications in the technical sense, but rather identity claims like 'Tully is Cicero' or 'Five feet is my height'. A general expression such as 'human' can appear both

${ }^{35}$ See Lowe, 2006, 21: 'The terms 'particular' and 'universal' themselves, we may say, do not strictly denote categories, however, because they are transcategorical, applying as they do to entities belonging to different basic categories'. 
as the subject and as the predicate of predicative assertions, as in 'A human is a vertebrate', and 'Cicero is a human'.

Taken together with the distinction between inhering and non-inhering entities, this yields a fourfold distinction of entities, the so-called ontological square (represented in Figure 4). ${ }^{36}$ Many ontologists accept only a selection of the fields of this ontological square. David Armstrong, for example, tries to manage with fields I and IV only, namely, particular substances and property universals (Armstrong, 1978 and 1997). Ontologists who see First-Order Logic on its standard reading as a tool for ontology arrive at the same result. The particulars correspond on this account to the individual constants (' $a$ ', ' $b$ ', ' $c$ ' $\ldots$ ), and the property universals correspond to the predicate variables (' $F$ ', ' $G$ ', ' $R$ ' ...). The view that the formula ' $F(a)$ ' is the key to ontology - that such formulae, along with relational expressions such as ' $R(a, b)$ ', in effect, form a mirror of reality - has been dubbed fantology by Smith (2005a).

Those philosophers who are prepared to allow events into their ontologies, such as Donald Davidson (1980), also accept continuants, which intimately resemble entities in field II. Russell, by contrast, wanted to completely eliminate the level of individuals, and to satisfy himself with fields III and IV, ${ }^{37}$ most likely having been influenced by Leibniz's theory of individual concepts. ${ }^{38}$ Nominalist philosophers, by contrast, accept only entities from the two lower fields, I and II. Some philosophers even try to make do with only one of these two categories. For example, the individual accidents in field II are the only basic entities for tropists; they call these abstract particulars or tropes, ${ }^{39}$ and see individual substances such as you and me as more or less loosely connected bundles of such tropes.

\footnotetext{
${ }^{36}$ See Smith, 2003a. On the history of such diagrams see Angelelli, 1967, 12; see also Wachter, 2000, 149. One of the most important contemporary representatives of a four-category ontology is E. J. Lowe; see in particular 2006.

37 See e.g. Russell, 1940, ch. 6; and 1948, Part II, ch. 3 und Part IV ch. 8; 1959, ch.9. For a similar position see Hochberg 1965, 1966, and 1969.

38 Russell (1948) attributes this conception explicitly to Leibniz. See also Armstrong, 1978, I 89: '[...] while the influence of Leibniz to Russell is clear, it is less clear that Leibniz held this theory of the nature of particulars

${ }^{39}$ Two classic presentations of this position can be found in Williams, 1953, and Campbell, 1990. See also Macdonald, 1998 and Trettin, 2000.
} 
Figure 4: Aristotle's Ontological Square

\begin{tabular}{|c|c|c|}
\hline & $\begin{array}{c}\text { Substantial } \\
\text { Not in a subject }\end{array}$ & $\begin{array}{c}\text { accidental, non-substantial } \\
\text { In a subject }\end{array}$ \\
\hline $\begin{array}{c}\text { universal, general } \\
\text { Predicated } \\
\text { of a subject }\end{array}$ & $\begin{array}{c}\text { III. } \\
\text { substance universals } \\
\text { Human being } \\
\text { Horse }\end{array}$ & $\begin{array}{c}\text { IV. } \\
\text { non-substance universals } \\
\text { Being white } \\
\text { Knowing }\end{array}$ \\
\hline $\begin{array}{c}\text { Individual } \\
\text { Not Predicated } \\
\text { of a subject }\end{array}$ & $\begin{array}{c}\text { individual substances } \\
\text { This human being } \\
\text { This horse }\end{array}$ & $\begin{array}{c}\text { This individual whiteness } \\
\text { This individual knowing } \\
\text { Indidual }\end{array}$ \\
\hline
\end{tabular}

Aristotle accepted all four cells of the ontological square, which he sees as, together, forming a transparent partition of reality. Thus, he reflects the commonsensical understanding of most people, according to which elements of all four fields exist. In daily life, we assume that George W. Bush (field I) exists as well as the species elephant (field III), the virtue of courage (field IV), and the individual white color of my skin, which ceases to exist at some time in summer, when my skin takes on a brown color instead (field II). Ontologists who want to get rid of one or more of these fields represent some kind of reductionist position. They must produce an alternative explanation for why we suppose in our everyday understanding that these things exist. They do this mainly through explaining our reference to entities in these fields as merely a roundabout way of talking about entities in other, more highly favored, fields.

There are some basic relations that obtain among entities in the four fields of the ontological square:

- Individual accidents inhere in individual substances.

- Non-substance universals characterize substance universals.

- Individual substances instantiate substance universals.

- Individual accidents instantiate accident universals.

- Individual substances exemplify accident universals. 
A picture of the world which did not provide a special place for occurrents would be incomplete. There are of course important relations that obtain between occurrents and continuants, for there are individual substances which take part in individual processes and events. We can thus expand the ontological square to an ontological sextet, which can be illustrated in Figure 5 (Smith, 2005a). The relations of inherence, exemplification, instantiation, and participation govern the relations among the entities in these four fields. They are important formal-ontological relations; regardless of which area of reality we want to represent, we must take all of these relations into account.

Figure 5: The Ontological Sextet and the Formal-ontological Relations

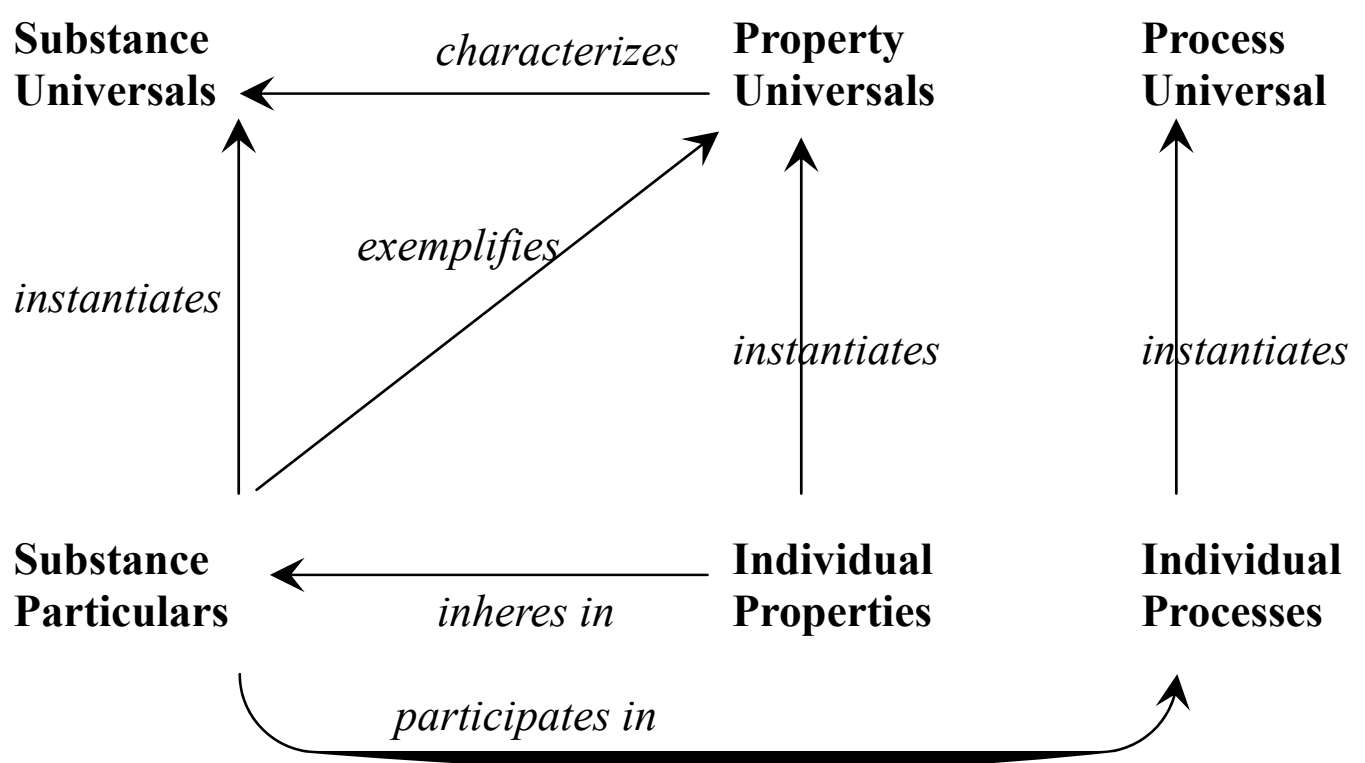

\section{Complex Entities}

In addition to the categories we have discussed thus far, discussions take place among modern ontologists about complex entities such as states of affairs, sets, mereological sums, and classes.

States of affairs are all of those complex entities which can be described with a 'that' sentence. That the ball is round and that the cat is on the mat are two examples of states of affairs. Both are complexes of entities falling among the various categories which we have just discussed. That a person is sick is a complex composed of a substance (this person), and a certain quality or disposition (sickness). The state of affairs that a certain molecule is attached to a receptor is composed of: a substance (the molecule), a part 
of a substance (the receptor), and the two-place relation of being attached. States of affairs can have other states of affairs as components. The state of affairs that the doctor has discovered that her patient has the flu is composed of the doctor, the intentional two-place relation of having discovered, and of the state of affairs that the patient has the flu. The thesis that all states of affairs are complex, or composite, entities seems to be called into question by expressions such as 'that it rains', which are constructed from impersonal pronouns such as 'it'. For these expressions cannot be divided linguistically into a predicate, on the one hand, and a referring subject expression, on the other. But this does not mean that the entities for which they stand cannot be analyzed ontologically. The state of affairs that it is raining is clearly composed of raindrops moving from place to place; thus, it is composed of a collective of movements undergone by a multiplicity of raindrops.

Sets are well known from mathematics. Sets are collections of elements. We say that sets contain elements as their members. And we say that certain entities are (or are not) elements of certain sets. The relation is an element of is represented by the sign ' $\in$ ', while the relation is not an element of by the sign ' $\notin$ '. In addition, set theorists discuss a range of relations between sets such as the intersection, the union, the subset relations, and the relation of set-theoretical difference. ${ }^{40}$ The intersection of two sets, for example, is the set - which may perhaps be empty - that contains as members exactly those entities which are members of both initial sets.

We can represent sets either extensionally, by listing their elements, or intensionally, by pointing to a feature common to all elements that is sufficient for set membership. Extensionally, sets usually are represented by means of lists whose elements are separated by commas and placed in closed parentheses. For example, the set of prime numbers less than 10 is $\{2,3,5,7\}$. But \{Aristotle, 2, my stethoscope\} is a set as well; thus, sets can be built out of arbitrarily designated elements. To be sure we can represent sets intensionally, without such a list, simply by specifying the characteristics that the elements belonging to them share and that are sufficient for set membership. Examples of this sort of description of a set would be 'the set of all patients at noon on the November 1, 2008 in Berlin', or 'the set of all such patients with a fever'. These sorts of descriptions are sometimes represented in the form: $\{\mathrm{x} \mid \mathrm{x}$ is a patient and

${ }^{40}$ For an overview see e.g. Bucher, 1998, Ch. 1. 
has a fever\}, which is read as 'the set of all things $\mathrm{x}$, such that: $\mathrm{x}$ is a patient and has a fever'. Additional examples of set descriptions are ' $\{x \mid x$ is round $\}$ ', and ' $\{\mathrm{x} \mid \mathrm{x}$ is red $\}$ '.

Sets are identical when they contain the same elements. The set description ' $\{2,3,5,7\}$ ' denotes the same set as the description 'the set of prime numbers less than 10', because each element contained in $\{2,3,5$, $7\}$ is also contained in the set of prime numbers less than 10 , and vice versa. The two sets, thus, are identical. From this criterion of identity, it follows that sets cannot survive the loss of any of their elements; the same set cannot have different elements at different points in time: different elements, different sets. From this criterion for set identity, it also follows that sets, in a certain sense, are timeless; hence, sets can include elements which exist at different times and at no times. They are also outside space (if the elements of a set move about in space the set is not affected in any way). It follows further that the order of the elements in a set is irrelevant. Thus:

$$
\{a, b\}=\{b, a\} .
$$

It also follows that repetitions of elements are irrelevant for set identity. Thus it holds that:

$$
\{a, a\}=\{a\}
$$

In order to know whether $\{\mathrm{x} \mid \mathrm{x}$ is red $\}$ and $\{\mathrm{x} \mid \mathrm{x}$ is round $\}$ are the same sets, we must know what sorts of things are available in the world, or in some specially selected universe of discourse. If the world consisted merely in a red circle, a yellow triangle, and a blue square, then these two set descriptions would indeed denote the same set; that is, the set red circle $\}$. In the actual world, there are circles that are not red and, therefore, according to the criterion for set identity in the actual world these two sets are not identical. The criterion for set identity also entails that there are no two distinct empty sets.

Because sets are independent of space and time, they count as abstract entities. The curly brackets are a sort of mechanism of abstraction: we take the names of concrete entities, place brackets around them, and create a name for something abstract. From 'Socrates', the name of the flesh-andblood Socrates who exists in space and time, we get ' $\{$ Socrates\}'; the name of an abstract entity, existing apart from space and time, that is the set 
composed of Socrates as its only element. Sets containing only one element are called singleton sets. The empty set itself, which plays a prominent role especially in mathematical explorations of the implications of the axioms of set theory, is referred to by means of the symbol ' $\varnothing$ '.

Sets can themselves be elements of other sets; and some sets have only sets as their members. There are also singletons of sets, and also the singleton of the empty set. Now this singleton can itself be an element of a set, for example of its singleton, and so forth. Thus the theory of sets sketched so far allows forming the singleton of the singleton of the singleton and so on of - the empty set. Hence it is possible to create potentially infinite structures out of nothing - more specifically, out of the empty set - and have these structures be isomorphic to the set of the natural numbers. Each of the following three rows fulfills the five Peano axioms for the natural numbers - only the interpretation of the neutral element 0 and the successor function are different:

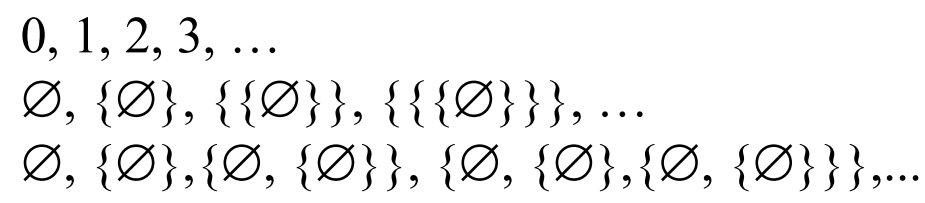

Since the singleton of a concrete thing is an abstract entity, the singleton and its only element must be distinct from one another. This is 'the mystery of the singletons: what distinguishes $a$ from $\{a\}$ '? (Simons, 2005, 145) The tricks that can be played with empty sets have induced some logicians and philosophers to seek an alternative to the set-theoretic view known as mereology (Simons, 1987; Ridder, 2002). Mereological sums are complexes which can be composed of various parts. My stomach, my sandwich, and the warmest corner of my office can comprise such a mereological sum. Just as with sets, there is virtually no limitation to the building of mereological sums. And just as with sets, many mereological sums (as in the example above) have a very artificial character. At any rate, very few mereological sums are natural wholes (though natural wholes such as organisms are among the most interesting of mereological sums). While sets are abstract entities even when composed of concrete elements, mereological sums composed of concrete elements are concrete things as well. Mereological sums exist in space and time, but only as long as all of their parts exist. A mereological sum does not survive the loss or destruction of one of its parts. Losing a part will result in another mereological sum. We speak of proper parts if we want to indicate that the 
putative part is not identical with the whole. A non-proper part can, analogously to non-proper subsets, also be identical with the whole.

In many ontologies, part-whole relations are used as formal-ontological relations. The theory of granular partitions (Chapter 6) introduces an approach which attempts to blaze a third trail between set theory and mereology, in order to link the concreteness of mereological sums with the hierarchical nature of the element-of relation.

Where sets can have members of arbitrarily different sorts, we shall use 'class' in what follows to refer to collections of members which are in some sense constrained, as for example in: the class of mammals, the class of red things, the class of positively charged electrons. The category of class thus represents an attempt to do away with the arbitrary nature of set construction. ${ }^{41}$ Although 'set' and 'class' are often used as synonyms, we will use them to signify different things, as for example in SUMO, where

'Set' is the ordinary set-theoretic notion, and it subsumes 'Class', which, in turn, subsumes 'Relation A'. 'Class' is understood as a 'Set' with a property or conjunction of properties that constitute the conditions for membership in the 'Class' (Niles and Pease 2001).

This also follows Smith, Kusnierczyk, Schober, and Ceusters $(2006,60)$ for whom 'class' signifies 'a collection of all and only the particulars to which a given general term applies'.

When the general term connected to a class represents a universal, we can speak of a natural class: a natural class is the totality of instances of a universal. Whereas sets may be constructed by means of enumeration, natural classes require that there be universals of which they are the extension. Two natural classes are identical if they represent the same universal. Because not all general expressions correspond to universals, not all classes are natural classes. These non-natural classes are called 'defined classes', like for example: the class of diabetics in London on a certain day, or the class of hospitals in San Diego.

Not every set, on this view, corresponds to a class. For example, \{Aristotle, 2, my stethoscope \} is a set constructed through the listing of its elements. However, it does not correspond to a natural class, for it is not the extension of any universal; nor does it correspond to any class at all,

\footnotetext{
${ }^{41}$ There are earlier attempts to link intensional elements with set theory; for example in Feibleman, 1974. The remarks presented here draw on Chapter 11 of this volume. See also Smith, et al., 2005, Smith, 2005.
} 
for there is no general expression (other than 'element of that set') under which precisely these three things fall. From a linguistic point of view we thus need, for the definition of a class, at least one general expression, whereas sets, such as the above example, can be denoted alone with proper names and definite descriptions.

Unlike set theory, class theory does not require us to know what things there are in the world in order to say that the class of red things and the class of round things are different from one another. And while there is only one empty set, there can be many different empty classes: for example, the class of all phlogiston, the class of all perpetual-motion machines, or the class of round squares. Since, however, they represent different universals, they are certainly different from one another. In addition, classes, but not sets, can survive the destruction or coming into existence of new instances; for sets are individuated by their elements, whereas natural classes are individuated by a universal which stays the same even as it has different instances at different times.

Figure 6: A Combination of Taxonomy and Partonomy ${ }^{42}$

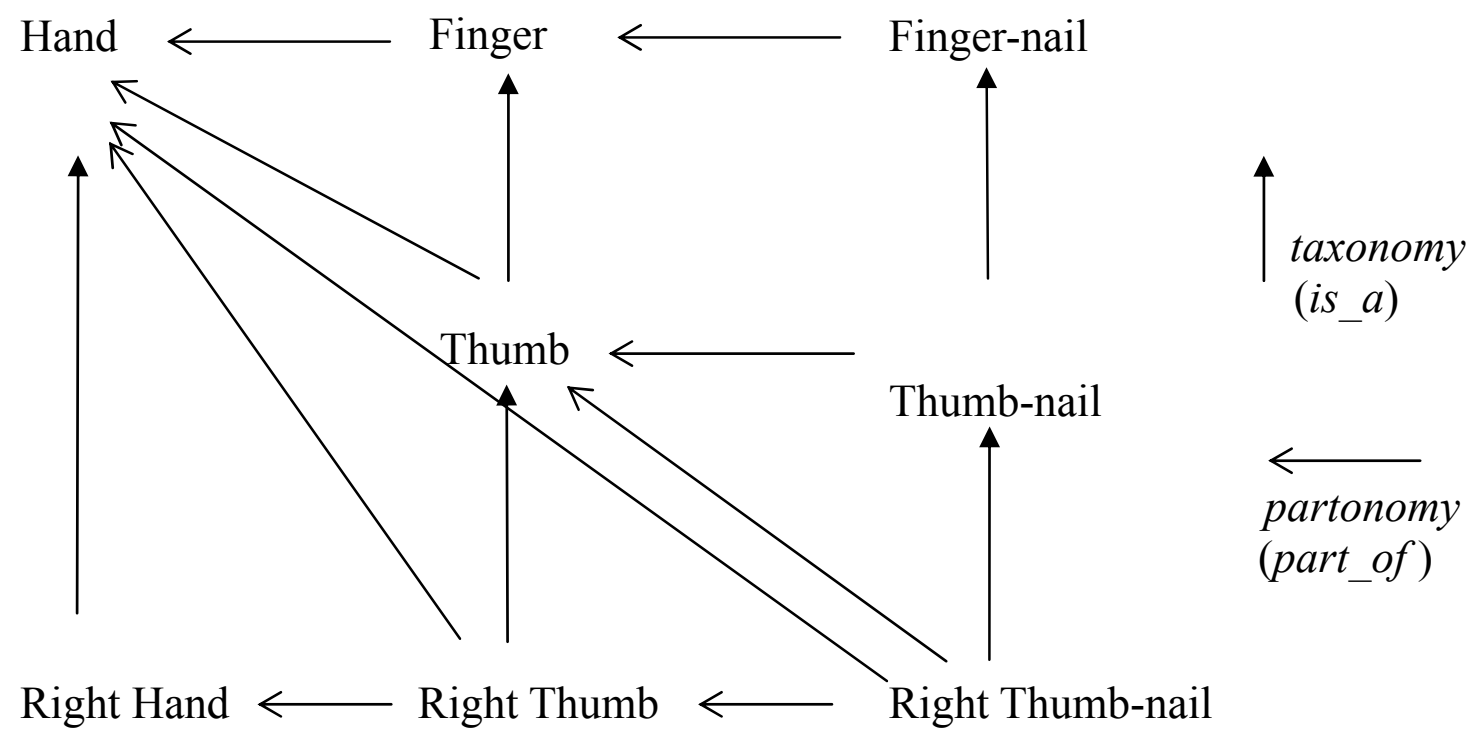

The result of dividing entities into classes is called a classification. Instead of speaking of a class we sometimes speak of a taxon (or, in the plural, of taxa, derived from the Greek word tattein, to place in order); we can speak, correspondingly, of a taxonomy. A taxonomy must be distinguished from a partonomy. While a classification or a taxonomy divides

${ }^{42}$ From Zaiss et al., 2005, 64. 
a universal into species or kinds, a partonomy divides a whole into its parts. It is particularly interesting to combine a partonomy with a classification, which has been done in Figure 6.

\section{The Unpolished Edges of the Sowa Diamond}

We are now equipped to look more closely at the Sowa diamond. Sowa sees his ontology as a melting pot of the process ontology of Whitehead and the triadic category theory of Charles Sanders Peirce. In light of what we have already seen in this chapter, however, we can point to some things that have gone badly wrong in this melting pot. The systematic presentation of Sowa's ontology comprises a combination of three distinctions:

- a dichotomy between Continuant and Occurrent

- a dichotomy between Physical and Abstract

- a trichotomy (which Sowa attributes to Peirce) between Independent, Relative, and Mediating.

A first point of criticism could be the question whether the dichotomy Physical vs. Abstract, and the Peirce-inspired trichotomy, are in fact appropriate means of classification. I will not discuss this question here. These two dichotomies and the trichotomy, taken together, yield twelve combinatorial possibilities, which I would like to examine more closely.

Figure 7: The Ten Central Categories of the Sowa Diamond from http://users.bestweb.net/ sowa/ontology/toplevel.htm (as of August 8, 2006)

\begin{tabular}{|c|c|c|c|c|}
\hline & \multicolumn{2}{|c|}{ Physical } & \multicolumn{2}{c|}{ Abstract } \\
\hline & Continuant & Occurrent & Continuant & Occurrent \\
\hline Independent & Object & Process & Schema & Script \\
\hline Relative & Juncture & Participation & Description & History \\
\hline Mediating & Structure & Situation & Reason & Purpose \\
\hline
\end{tabular}

In contrast to Sowa, I do not find all of these combinations of di- and trichotomies well advised. For example, there are no abstract occurrents (see Guarino, 2001): what occurs is never abstract. Although there are universals that are instantiated by occurrents and only by occurrents, these universals are themselves not temporally extended entities and thus they 
are not themselves occurrents (compare Chapter 12). To name an additional example: from our Aristotelian point of view, the category Object is the only one found among independent entities: all occurrents and all abstract entities are necessarily ontologically dependent entities.

Other combinatorial possibilities, like Mediation and Participation, seem to correspond more closely to what we would see as relations between categories than as categories in themselves. Description and History, by contrast, can both be understood as linguistic entities that are not distinguished ontologically, but rather by means of their objects. A description does not become an occurrent simply by being a description of an occurrent. Analogously, a Purpose does not become an occurrent simply because it aims at the realization of an occurrent (and even this does not hold for all purposes). Just as little is the general schema or recipe that describes how, e.g., an operation proceeds (what Sowa calls the Script of this event) thereby itself an occurrent. This is particularly clear when Sowa introduces a sheet of music and series of pictures on a roll of film as examples of scripts, as these exist in space and time and are thus, according to Sowa's own definition, physical entities and not abstract.

Sowa has designed his diamond in such a way that he characterizes the various options of his di- and trichotomies by means of axioms such that the central categories coming about through a combination of these options inherit the axioms of the options constituting them. Because of the problems just discussed it does not come as a surprise that this does not work. For example, Sowa characterizes occurrents inter alia as having sequential temporal phases and participants as spatial parts. The category Reason, which is characterized by Sowa as a mediating abstract occurrent, is meant to inherit these axioms. But reasons neither have temporal phases nor participants as spatial parts. Thus the principle of construction underlying the diamond cannot be held up.

An additional problem with Sowa's suggestion is that - notwithstanding its systematic outlook - it fails to encompass all entities. For example, he characterizes the expression 'physical' (which is for him primitive) by saying that everything that is physical exists in a certain place and at a certain time. But places and times, over which he quantifies in the corresponding axioms, do not themselves appear in the diamond, and it is hard to see how they can be integrated in the uncompromising architecture of Sowa's system. They would seem to have a place next to the diamond, not within it. And even if physics has not yet encompassed space and time 
in a Grand Unified Theory, it is indispensable for the ontologist to capture such important categories in his system.

\section{Conclusion}

Our criticisms of OpenCyc and the Sowa Diamond show that the suggestions proffered within the fields of informatics and knowledge representation for the formation of a top-level ontology are not always satisfactory. In drawing on Aristotle's list of categories, in this chapter I have developed suggestions for a top-level ontology that corresponds to the basic characteristics of Basic Formal Ontology (BFO). The three ontological dichotomies of dependent versus independent, continuant versus occurrent, and universal versus particular, form an armory of categories that, by means of further distinctions, can be built upon and refined. In fact, BFO is already being used, in applications, by a number of biomedical ontology groups, many of which are members of the OBO Foundry (see Chapter 1). 